Chang-Seok Ki • Won Yong Lee • Do Hoon Han

Duk Hyun Sung • Kyung-Bok Lee • Kyung-A Lee

Sang Seon Cho - Seunghee Cho $\cdot$ Hyokkee Hwang

Kwang Min Sohn • Yeun Joo Choi · Jong-Won Kim

\title{
A novel missense mutation (I344K) in the SPG4 gene in a Korean family with autosomal-dominant hereditary spastic paraplegia
}

Received: February 20, 2002 / Accepted: May 21, 2002

\begin{abstract}
Hereditary spastic paraplegia (HSP) is a group of clinically and genetically heterogeneous neurodegenerative disorders characterized by slowly progressive spasticity and weakness of the lower extremities. Among eight loci linked with autosomal-dominant (AD)-HSP, the SPG4 locus on chromosome 2 p 22 accounts for about $40 \%$ of all patients. Recently, mutations in a new member of the AAA protein family, called spastin, have been identified as responsible for SPG4-linked AD-HSP. Here, we describe a novel missense mutation (c.1031T>A; I344K) in exon 7 of the SPG4 gene identified in a Korean family with typical clinical features of pure AD-HSP. The mutation affects the third amino acid of the highly conserved AAA cassette domain, which is the most fore part of the domain altered by a missense mutation reported so far. Clinical presentations of affected individuals carrying the I344K mutation were not different from those of pure AD-HSP with SPG4 mutations reported previously. However, it is noteworthy that neither urinary dysfunction nor involvement of upper extremities was noticed in this family. To our knowledge, this is the first report of genetically confirmed AD-HSP in Korea.
\end{abstract}

C.-S. Ki $\cdot$ K.-A Lee $\cdot$ J.-W. Kim ${ }^{1}(\square)$

Department of Clinical Pathology, Sungkyunkwan University School of Medicine, Samsung Medical Center, \#50 Ilwon-Dong, Kangnam$\mathrm{Gu}$, Seoul 135-710, Korea

Tel. +82-2-3410-2705; Fax +82-2-3410-2719

e-mail: jwonk@smc.samsung.co.kr

W.Y. Lee ${ }^{1} \cdot$ D.H. Han

Department of Neurology, Sungkyunkwan University School of Medicine, Samsung Medical Center, Seoul, Korea

\section{D.H. Sung}

Department of Physical Medicine and Rehabilitation, Sungkyunkwan University School of Medicine, Samsung Medical Center, Seoul,

Korea

K.-B. Lee

Department of Neurology, Daejon Military Hospital, Daejon, Korea

S.S. Cho $\cdot$ S. Cho $\cdot$ H. Hwang $\cdot$ K.M. Sohn $\cdot$ Y.J. Choi

Center for Clinical Research, Samsung Biomedical Research

Institute, Seoul, Korea

${ }^{1}$ These two authors contributed equally to this work.
Key words Autosomal dominant hereditary spastic paraplegia $\cdot S P G 4 \cdot$ Korean $\cdot$ Missense mutation $\cdot$ Spastin $\cdot$ AAA cassette domain

\section{Introduction}

Familial or hereditary spastic paraplegia (HSP) is a group of clinically and genetically heterogeneous neurodegenerative disorders that share the primary features of slowly progressive spasticity and weakness of the lower limbs. Traditionally, HSP has been classified on the basis of the inheritance pattern, the age of onset, and the presence (complicated HSP) or absence (pure HSP) of additional neurological abnormalities such as mental retardation, dementia, ataxia, optic neuropathy, retinopathy, and so on. Although both forms can be inherited in an autosomal-dominant (ADHSP), autosomal-recessive (AR-HSP) or X-chromosomelinked (X-HSP) manner, pure AD-HSP is the most common form of HSP (Fink et al. 1996; Harding 1983; Tallaksen et al. 2001).

To date, seven genetic loci for pure AD-HSP have been mapped: chromosomes 14q (SPG3), 2p (SPG4), 15q $(S P G 6), 8 \mathrm{q}(S P G 8), 12 \mathrm{q}(S P G 10), 19 \mathrm{q}(S P G 12)$, and 2q (SPG13). Among these loci, SPG4 is the most common and represents approximately $40 \%$ or more of pure $\mathrm{AD}$ HSP cases (Casari and Rugarli 2001; Fink et al. 1996; Tallaksen et al. 2001). Recently, Hazen et al. (1999) identified the spastin (SPG4) gene, which encodes a new member of the AAA (ATPase associated with diverse cellular activities) protein family. After this discovery, more than 70 mutations of the SPG4 gene have been reported, including missense, nonsense, and splice-site mutations, as well as insertions and deletions (Bürger et al. 2000; de Bantel et al. 2001; Fonknechten et al. 2000; Hazan et al. 1999; Hentati et al. 2000; Jiggins et al. 2001; Lindsey et al. 2000; Namekawa et al. 2001; Santorelli et al. 2000; Svenson et al. 2001). Here, we present a novel missense (I344K) mutation in the SPG4 gene in a large Korean family with pure AD-HSP. 
Fig. 1. Pedigree of a Korean family with autosomal-dominant hereditary spastic paraplegia (AD-HSP) carrying a missense mutation (I344K) of the SPG4 gene

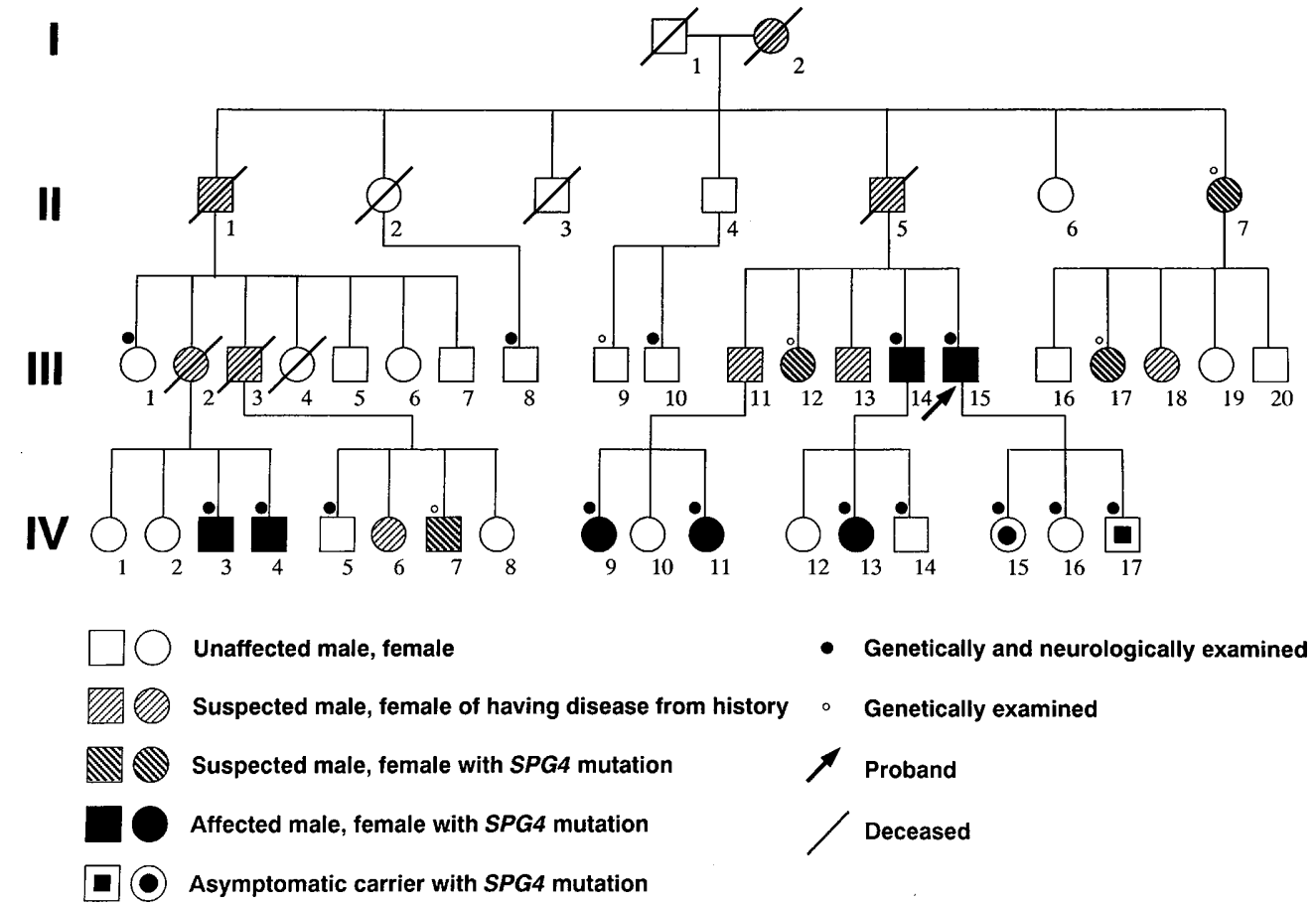

\section{Subjects and methods}

\section{Case report}

A 40-year-old male visited the neurology department complaining of weakness and spasticity in the lower extremities. He first noted stiffness of both legs and gait unsteadiness at age 33, and it had progressed over the years. His father and all of his siblings as well as many relatives on his father's side had similar problems (Fig. 1). On neurological examination, his gait was spastic but independent. He was well oriented and had normal cognitive function without evidence of decreased visual acuity, optic neuropathy, retinopathy, pigmental macular degeneration, nystagmus, or dysarthria. Deep tendon reflex, muscle strength, tone, sensation, and coordination of upper extremities were normal. The lower extremity showed proximal and distal weakness, marked increase in tone, pathologically brisk reflexes, ankle clonus, and extensor plantar reflex, but normal sensation.

\section{Clinical evaluation}

After we had obtained informed consent, a detailed neurological examination including evaluation of the cranial nerves, deep tendon reflexes, motor, and sensory systems was performed on 14 additional family members of the proband. Information on deceased family members as well as on other family members who were not evaluated neurologically was obtained from the proband and other senior members of the family. Subjects were classified into four groups: (1) "definitely affected" if they had spasticity, increased reflexes, and extensor plantar response; (2) "prob- ably affected" if they had only increased reflexes or extensor plantar response; (3) "possibly affected" if they had brisker reflexes in the lower limbs compared with the upper limbs; and (4) "normal" if they had no neurological abnormalities, as described previously (Fonknechten et al. 2000). Disability was assessed on a five-point scale: 1 , normal gait or very slight stiffness in the legs; 2 , moderate gait stiffness; 3 , unable to run but able to walk alone; 4, walk with help; 5 , wheelchair bound (Fonknechten et al. 2000).

\section{Molecular genetic study}

All 15 neurologically examined and 5 additional family members ( 4 were suspected to be affected and 1 was normal, based on the histories) were included in the molecular genetic study, after we obtained informed consent. Genomic DNA was isolated from peripheral blood leukocytes by using a Wizard Genomic DNA Purification kit following the manufacturer's instruction (Promega, Madison, WI, USA). Since SPG4 is the most common cause of pure ADHSP and because it was the only locus with an identified gene, we decided to perform direct sequencing analysis of the SPG4 gene [during preparation of this article, however, Zhao et al. (2001) identified a second gene, $S P G 3 A$, causing early-onset AD-HSP]. By using the proband's DNA, all 17 exons of the SPG4 gene were amplified by polymerase chain reaction (PCR), as described previously (Hazan et al. 1999). Cycle sequencing was performed with a BigDye Terminator Cycle Sequencing Ready Reaction kit, version 2.0. (Applied Biosystems, Foster City, CA, USA) on an automated ABI Prism 3100 genetic analyzer (Applied Biosystems). 

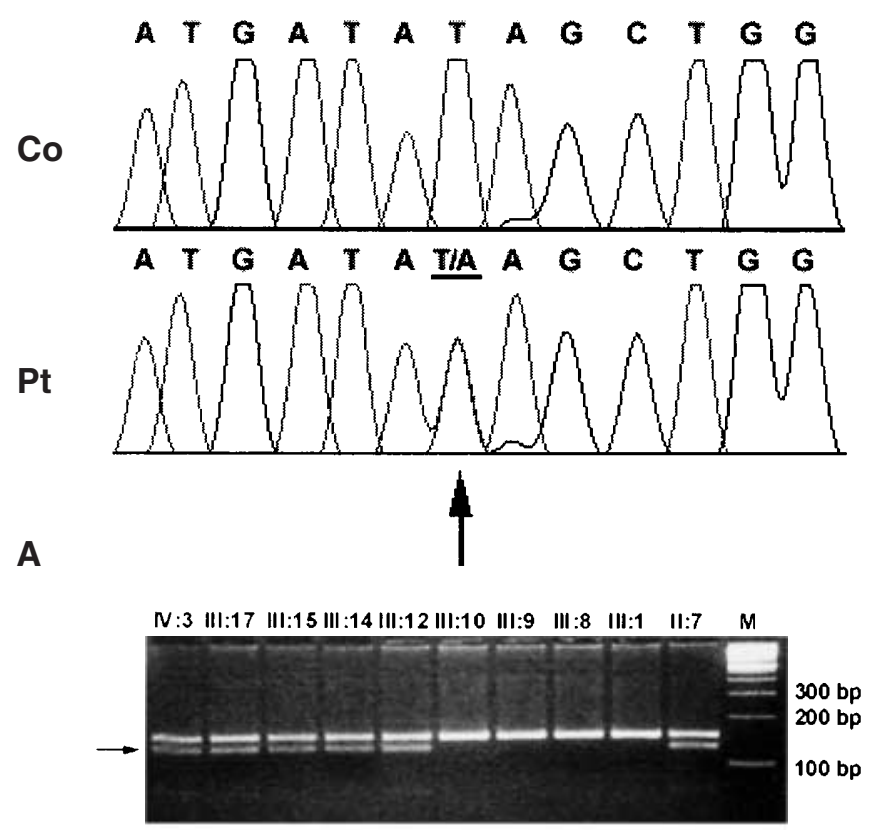

$N: 17$ IV:16 N:15 IV:14 N:13 N:11 IV:9 $N: 7$ N:5 IV:4 $M$

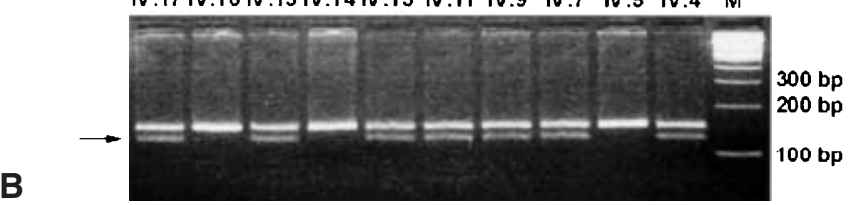

Fig. 2A,B. Identification of the SPG4 gene mutation. Direct sequencing analysis demonstrated a heterozygous $\mathrm{T}$ to $\mathrm{A}$ transversion in exon 7 of the SPG4 gene (c.1013T $>$ A), resulting in an $1344 \mathrm{~K}$ missense mutation (A; arrow). By the mismatch polymerase chain reactionrestriction fragment length polymorphism (PCR-RFLP) method, a 152-bp PCR product is divided into 129 and $23 \mathrm{bp}$ (not visible) fragments only with the mutant allele present (B; arrow). All affected family members (II:7, III:12, III:14, III:15, III:17, IV:3, IV:4, IV:7, IV:9, IV:11, and IV:13; refer to Fig. 1.) as well as two asymptomatic offspring of the proband (IV:15 and IV:17) had the mutant allele, whereas unaffected members (III:1, III:8, III:9, III:10, IV:5, IV:14, and IV:16) did not. $C o$, control; $P t$, patient; $M, 100$-bp size marker

After finding a missense mutation in exon 7 of the SPG4 gene, a mismatch PCR-restriction fragment length polymorphism (RFLP) method was applied to confirm it. Since the mutation neither created nor destroyed a restriction site, we designed a mismatch primer (5'-ggaacagctg $\underline{G}$ taaatttgatg $\underline{T t a}-3^{\prime}$; underlined sequences indicate mismatched sites) to introduce a recognition sequence (ttaa) for MseI in the amplified DNA. The reverse primer was $5^{\prime}$-actactatggattcagtaacagatgg-3'. With this method, a 152-bp PCR product is divided into 129- and 23bp fragments only with the mutant allele present, when treated with MseI (Fig. 2).

\section{Results}

Clinical findings

Clinical features of affected individuals are summarized in Table 1 . Out of 15 family members examined neurologi- cally, 7 subjects were clinically affected. Among them, 2 subjects (III:14 and III:15) were classified as "definitely affected" because they had all of spasticity, hyperreflexia, and extensor plantar reflex. The others (IV:3, IV:4, IV:9, IV:11, and IV:13) were classified as "probably affected." Interestingly, 3 of the "probably affected" members (IV:3, IV:9, and IV:13) were unaware of any symptoms in the lower extremities. Only 1 subject (III:14) had sensory impairment of vibration and position in the lower extremities. None had cognitive dysfunction, cerebellar ataxia, parkinsonism, skin lesion, peripheral neuropathy, epilepsy, dysarthria, dystonia, urinary urgency, constipation, pes cavus, or visual symptoms. Age at onset was variable between 14 and 39 years (mean $\pm \mathrm{SD}, 28 \pm 10.7$ years), and the initial symptom was the insidious appearance of stiffness in the legs for all the affected members. Regarding disability status, all the clinically affected subjects except the proband (III:15) were rated as stage 1 . The proband was in stage 3 because he was unable to run but was able to walk alone.

\section{Molecular genetic findings}

By mutation analysis, we found a heterozygous $\mathrm{T}$ to $\mathrm{A}$ transversion in exon 7 of the SPG4 gene (c.1031T $>$ A), which alters the amino acid sequence from Ile to Lys at codon 344 (I344K; Fig. 2). All seven clinically affected family members had the missense mutation. Other family members with normal neurological findings did not have the mutation, except for two (IV:15 and IV:17), who were 15 and 8 years old (Fig. 1). They were thought to be asymptomatic mutation carriers in whom neurological signs of HSP had not developed at the time of the examination. Of interest, IV:15 had the subjective symptom of slight stiffness in the lower legs but showed normal neurological findings.

Among the five additional family members (II:7, III:9, III:12, III:17, and IV:7) tested for the mutation, the same heterozygous I344K mutation was found in all four individuals suspected to be affected from their history (II:7, III:12, III:17, and IV:7), but not in the one normal member (III:9). We confirmed the mutation by the mismatch PCRRFLP method described above (Fig. 2), and the mutation was not found in 100 normal chromosomes.

\section{Discussion}

Up to now, more than 70 mutations of the SPG4 gene have been described. Missense, nonsense, and splice-site mutations, as well as deletions and insertions, have all been observed. Although these mutations have been found scattered along the length of $S P G 4$, the majority of the missense mutations are localized between exons 7 and 16 (Bürger et al. 2000; Fonknechten et al. 2000; Hazan et al. 1999; Hentati et al. 2000; Lindsey et al. 2000; Svenson et al. 2001). This region of SPG4 has been shown to correspond to a highly conserved ATPase domain, also called the AAA cassette, 
Table 1. Neurological findings in seven family members with the SPG4 mutation

\begin{tabular}{|c|c|c|c|c|c|c|c|}
\hline Patients & III:14 & III: $15^{\mathrm{a}}$ & IV:3 & IV:4 & IV:9 & IV:11 & IV:13 \\
\hline Age at examination (years) & 44 & 41 & 34 & 31 & 28 & 17 & 14 \\
\hline Age at onset (years) & 39 & 33 & Unaware & 26 & Unaware & 14 & Unaware \\
\hline \multicolumn{8}{|l|}{ Lower extremities } \\
\hline Weakness & - & + & - & + & - & \pm & - \\
\hline Hyperreflexia & + & + & + & + & + & + & + \\
\hline Spasticity & + & + & + & + & - & + & + \\
\hline Sensory ataxia & + & - & - & - & - & - & - \\
\hline Ankle clonus & + & + & + & + & - & - & - \\
\hline Extensor plantar reflex & + & + & - & - & - & - & - \\
\hline Sensory impairment & + & - & - & - & - & - & - \\
\hline \multicolumn{8}{|l|}{ Upper extremity } \\
\hline Hyperreflexia & - & - & - & - & - & - & - \\
\hline Spasticity & - & - & - & - & - & - & - \\
\hline Sensory ataxia & - & - & - & - & - & - & - \\
\hline Sensory impairment & - & - & - & - & - & - & - \\
\hline Disability stage $^{c}$ & 1 & 3 & 1 & 1 & 1 & 1 & 1 \\
\hline
\end{tabular}

\footnotetext{
${ }^{a}$ Proband

${ }^{\mathrm{b}}$ Age at onset was estimated as the time when symptoms were first noted by the patient

${ }^{\mathrm{c}}$ Disability stage: 1 , normal gait or very slight stiffness in the legs; 2 , moderate gait stiffness; 3 , unable to run but able to walk alone; 4 , walk with help; 5 , wheelchair bound
}

which is located between amino acids 342 and 599 of spastin (Hazan et al. 1999). The importance of the AAA cassette in spastin function is suggested by its high degree of conservation among AAA protein family members and by the locations of the spastin gene mutations identified to date (Hazan et al. 1999; Svenson et al. 2001).

The mutation described in the present study is also localized in exon 7 of the SPG4 gene, affecting the third amino acid of the highly conserved AAA cassette, which is the most fore part of the domain altered by a missense mutation reported so far. According to the multiple alignment results of eight proteins exhibiting high homology to human and mouse spastin, codon 344 is relatively well conserved in that only branched amino acids such as Ile and Val were observed (Hazan et al. 1999). The missense mutation I344K replaces Ile, a hydrophobic aliphatic amino acid, with Lys, a polar basic amino acid. Therefore, it seems likely that this change would severely reduce or abolish the function of the spastin protein (Bürger et al. 2000; Lindsey et al. 2000).

The exact function of spastin is still not known. However, it has been found that the level of spastin mRNA tested in tissues from patients with SPG4 mutations is uniformly reduced. Therefore, the presumed loss of protein function by inactivation of the AAA domain because of different types of mutations in the SPG4 gene may be the possible pathogenic mechanism of HSP (Bürger et al. 2000; Fonknechten et al. 2000; Hazan et al. 1999; Lindsey et al. 2000).

According to the recent review by Tallaksen et al. (2001), the major clinical findings of 224 SPG4 mutation carriers are (1) almost $25 \%$ of gene carriers are asymptomatic; (2) the mean age at onset is 29 years, but ranges from the first year of life to 74 years; (3) disease progression is highly variable; (4) associated signs increase in frequency and/or severity with the duration of the disease; and (5) there is no apparent correlation between the type of mutation (missense versus truncating) and the clinical features.

Clinical findings of affected individuals presented in this report are similar to those described above. In our study, 2 $(15.4 \%)$ out of 13 family members carrying the $1344 \mathrm{~K}$ missense mutation were neurologically free. This percentage of asymptomatic carriers was somewhat lower than that reported in previous studies (Fonknechten et al. 2000; McMonagle et al. 2000; Tallaksen et al. 2001). But this proportion could be increased to $38.5 \%$ if we added the three subjects who were not aware of any symptoms or signs. In our patients, mean age at onset was 28 years, which is consistent with previous data. Wide variation of disease progression was also noted.

It is noteworthy that the upper extremities as well as urinary function were relatively preserved in this family. In addition, sensory impairment was found in only one subject (III:14). All these features are common in AD-HSP patients carrying the SPG4 mutation. About $73 \%$ of patients with AD-HSP have hyperreflexia in the upper extremities, and $38 \%-59 \%$ of AD-HSP patients may be affected by urinary frequency, urgency, or hesitancy. Furthermore, sensory impairment, especially abnormal vibration sensations in the lower limb, is also a common presentation, found in $58 \%-68.2 \%$ of all AD-HSP patients with the SPG4 mutation (Fonknechten et al. 2000; McMonagle et al. 2000).

In summary, we have identified a novel missense mutation in the SPG4 gene in a Korean family with pure ADHSP. The clinical findings described in the present study may be useful in future genotype-phenotype correlation studies. To the best of our knowledge, this is the first report of genetically confirmed pure AD-HSP in Korea.

Acknowledgments This work was supported by funds from the Center for Functional Analysis of Human Genome, Korea. 


\section{References}

Bürger J, Fonknechten N, Hoeltzenbein M, Neumann L, Bratanoff E, Hazan J, Reis A (2000) Hereditary spastic paraplegia caused by mutations in the SPG4 gene. Eur J Hum Genet 8:771-776

Casari G, Rugarli E (2001) Molecular basis of inherited spastic paraplegias. Curr Opin Neurol 11:336-342

de Bantel A, McWilliams S, Auysh D, Echol C, Sambuughin N, Sivakumar K (2001) Novel mutation of the spastin gene in familial spastic paraplegia. Clin Genet 59:364-365

Fink JK, Heiman-Patterson T, Bird T, Cambi F, Dube MP, Figlewicz DA, Fink JK, Haines JL, Heiman-Patterson T, Hentati A, PericakVance MA, Raskind W, Rouleau GA, Siddique T, the Hereditary Spastic Paraplegia Working Group (1996) Hereditary spastic paraplegia: advances in genetic research. Neurology 46:1507-1514

Fonknechten N, Mavel D, Byrne P, Davoine CS, Cruaud C, Boentsch D, Samson D, Coutinho P, Hutchinson M, McMonagle P, Burgunder JM, Tartaglione A, Heinzlef O, Feki I, Deufel T, Parfrey N, Brice A, Fontaine B, Prud'homme JF, Weissenbach J, Durr A, Hazan J (2000) Spectrum of SPG4 mutations in autosomal dominant spastic paraplegia. Hum Mol Genet 9:637-644

Harding AE (1983) Classification of the hereditary ataxia and paraplegias. Lancet 1:1151-1155

Hazan J, Fonknechten N, Mavel D, Paternotte C, Samson D, Artiguenave F, Davoine CS, Cruaud C, Durr A, Wincker P, Brottier P, Cattolico L, Barbe V, Burgunder JM, Prud'homme JF, Brice A, Fontaine B, Heilig B, Weissenbach J (1999) Spastin, a new AAA protein, is altered in the most frequent form of autosomal dominant spastic paraplegia. Nat Genet 23:296-303

Hentati A, Deng HX, Zhai H, Chen W, Yang Y, Hung WY, Azim AC, Bohlega S, Tandan R, Warner C, Laing NG, Cambi F, Mitsumoto H, Roos RP, Boustany RM, Ben Hamida M, Hentati F, Siddique T
(2000) Novel mutation in spastin gene and absence of correlation with age at onset of symptoms. Neurology 55:1388-1390

Jiggins JJ, Loveless JM, Goswami S, Nee LE, Cozzo C, De Biase A, Rosen DR (2001) An atypical intronic deletion widens the spectrum of mutations in hereditary spastic paraplegia. Neurology 56:14821485

Lindsey JC, Lusher ME, McDermott CJ, White KD, Reid E, Rubinsztein DC, Bashir R, Hazan J, Shaw PJ, Bushby KM (2000) Mutation analysis of the spastin gene (SPG4) in patients with hereditary spastic paraparesis. J Med Genet 37:759-765

McMonagle P, Byrne PC, Fitzgerald B, Webb S, Parfrey NA, Hutchinson M (2000) Phenotype of AD-HSP due to mutations in the SPAST gene: comparison with AD-HSP without mutations. Neurology 55:1794-1800

Namekawa M, Takiyama Y, Sakoe K, Shimazaki H, Amaike M, Niijima K, Nakano I, Nishizawa M (2001) A large Japanese SPG4 family with a novel insertion mutation of the $S P G 4$ gene: a clinical and genetic study. J Neurol Sci 185:63-68

Santorelli FM, Patrono C, Fortini D,Tessa A, Comanducci G, Bertini E, Pierallini A, Amabile GA, Casali C (2000) Intrafamilial variability in hereditary spastic paraplegia associated with an SPG4 gene mutation. Neurology 55:702-705

Svenson IK, Ashley-Koch AE, Gaskell PC, Riney TJ, Cumming WJ, Kingston HM, Hogan EL, Boustany RM, Vance JM, Nance MA, Pericak-Vance MA, Marchuk DA (2001) Identification and expression analysis of spastin gene mutations in hereditary spastic paraplegia. Am J Hum Genet 68:1077-1085

Tallaksen CME, Dürr A, Brice A (2001) Recent advances in hereditary spastic paraplegia. Curr Opin Neurol 14:457-463

Zhao X, Alvarado D, Rainier S, Lemons R, Hedera P, Weber CH, Tukel T, Apak M, Heiman-Patterson T, Ming L, Bui M, Fink JK (2001) Mutations in a newly identified GTPase gene cause autosomal dominant hereditary spastic paraplegia. Nat Genet 29:326-331 